\title{
Synchronized flow and wide moving jams from balanced vehicular traffic
}

\author{
Florian Siebel and Wolfram Mauser \\ Department of Earth and Environmental Sciences, \\ University of Munich, Luisenstraße 37, D-80333 Munich, Germany
}

(Dated: September 12, 2018)

\begin{abstract}
Recently we proposed an extension to the traffic model of Aw, Rascle and Greenberg. The extended traffic model can be written as a hyperbolic system of balance laws and numerically reproduces the reverse $\lambda$ shape of the fundamental diagram of traffic flow. In the current work we analyze the steady state solutions of the new model and their stability properties. In addition to the equilibrium flow curve the trivial steady state solutions form two additional branches in the flow-density diagram. We show that the characteristic structure excludes parts of these branches resulting in the reverse $\lambda$ shape of the flow-density relation. The upper branch is metastable against the formation of synchronized flow for intermediate densities and unstable for high densities, whereas the lower branch is unstable for intermediate densities and metastable for high densities. Moreover, the model can reproduce the typical speed of the downstream front of wide moving jams. It further reproduces a constant outflow from wide moving jams, which is far below the maximum free flow. Applying the model to simulate traffic flow at a bottleneck we observe a general pattern with wide moving jams traveling through the bottleneck.
\end{abstract}

PACS numbers: $89.40 . \mathrm{Bb}, 05.10 .-\mathrm{a}, 47.20 . \mathrm{Cq}$

\section{INTRODUCTION}

Modeling vehicular traffic flow using methods from continuum fluid dynamics has a long history [1, 2, 3, 4, 5], with many contributions mainly from traffic engineers, physicists and mathematicians. The existing models can be subdivided into first and higher order models according to the highest derivative appearing in the partial differential equations describing traffic flows 6]. 25] First order models, as the classical model of Lighthill, Whitham and Richards [7, 8], approximate the higher order models by neglecting diffusion terms in the same way the Euler equation approximates the Navier-Stokes equation. As a consequence, discontinuous data can develop, which have to be dealt with by a numerical algorithm.

In the existing literature on first order systems the role of a source term in the partial differential equations describing vehicular traffic flow on a road section without entries and exits has not been studied in depth. Instead, the main focus has been laid on the principal part of the equations [9], i.e. the collection of terms in the partial differential equation containing derivatives of order equal to the order of the partial differential equation 10 , and systems with constant relaxation time 11, 12, 13, 14, 15]. In [16], we presented the balanced vehicular traffic model (BVT model), which generalizes the model of Aw, Rascle and Greenberg [9, 11] by prescribing a more general source term subsumed under an effective relaxation coefficient. Unlike in earlier studies, this effective relaxation coefficient depends on both traffic density and velocity. As we showed in numerical simulations in 16], the model can reproduce the observed reverse $\lambda$ shape of the fundamental diagram of traffic flow 17].

In the current work we aim at obtaining a better understanding of the numerical results of the BVT model. In particular we study the appearance of the new branches in the fundamental diagram in the congested regime, which finally form the reverse $\lambda$ shape. Our explanation of the reverse $\lambda$ shape differs from earlier explanations, which explained the reverse $\lambda$ as a part of a single equilibrium flow branch. In contrast, as we will show, the (meta-) stable curve sections of three steady state branches form the reverse $\lambda$ in the BVT model. Hence, the criticism of the one-dimensionality of steady states of fluid-dynamical models [5] does not apply to the BVT model.

In the BVT model traffic flow is described by the following system of balance laws determining the density $\rho=\rho(t, x)$ and velocity $v=v(t, x)$ of vehicles

$$
\begin{aligned}
\frac{\partial \rho}{\partial t}+\frac{\partial(\rho v)}{\partial x} & =0, \\
\frac{\partial(\rho(v-u(\rho))}{\partial t}+\frac{\partial(\rho v(v-u(\rho)))}{\partial x} & = \\
\beta(\rho, v) \rho(u(\rho)-v) . &
\end{aligned}
$$

As usual, $(t, x)$ denote the time and space variable. $u(\rho)$ denotes the equilibrium velocity, which fulfills

$$
\begin{aligned}
& u^{\prime}(\rho)<0 \text { for } 0<\rho \leq \rho_{m}, \\
& \frac{d^{2}(\rho u(\rho))}{d \rho^{2}}<0 \text { for } 0<\rho \leq \rho_{m} .
\end{aligned}
$$

The effective relaxation coefficient $\beta(\rho, v)$ fulfills

$$
\begin{gathered}
\beta(\rho, v)<0 \text { for } 0<\rho_{1}<\rho<\rho_{2} \leq \rho_{m}, v=u(\rho), \\
\beta(\rho, v) \geq 0 \text { for } 0 \leq \rho \leq \rho_{1} \text { or } \rho_{2} \leq \rho \leq \rho_{m}, v=u(\rho) \\
\lim _{v \rightarrow 0, u_{m}=u(0)} \beta(\rho, v) \geq 0
\end{gathered}
$$

Note that in moving observer coordinates, Eqn. (2) reduces to

$$
\frac{d}{d t}(v-u(\rho))=-\beta(\rho, v)(v-u(\rho))
$$


i.e. $\beta$ can be interpreted as decay parameter. As an effective parameter, which takes into account the actual relaxation time but also the reaction time, the parameter can become negative for intermediate to high densities (5) (see [16]). Note that for $\beta<0$, the average velocity $v$ departs from the equilibrium velocity $u(\rho)$, i.e. $|v-u(\rho)|$ increases with time. For $\beta>0$, drivers approach the equilibrium velocity $u(\rho)$ with a rate determined by the value of $\beta$.

In [16] we used parameter functions and values which describe traffic flow only qualitatively. In the current work we use the equilibrium velocity of Newell [18]

$$
u(\rho)=u_{m}\left(1-\exp \left(-\frac{\lambda}{u_{m}}\left(\frac{1}{\rho}-\frac{1}{\rho_{m}}\right)\right)\right)
$$

with parameter values $u_{m}=160 \mathrm{~km} / \mathrm{h}, \lambda=$ 3600 [1/h/lane], $\rho_{m}=160[1 / \mathrm{km} /$ lane $]$ and an effective relaxation coefficient

$$
\begin{gathered}
\beta(\rho, v)= \begin{cases}\frac{a_{c}}{u-v}, & \text { if } \tilde{\beta}(\rho, v)(u(\rho)-v)-a_{c} \geq 0 \\
\frac{d_{c}}{u-v}, & \text { if } \tilde{\beta}(\rho, v)(u(\rho)-v)-d_{c} \leq 0 \\
\tilde{\beta}(\rho, v), & \text { else, }\end{cases} \\
\tilde{\beta}(\rho, v)=\frac{1}{\hat{T} u_{m}}\left(\left|u(\rho)-v+\alpha_{1} \Delta v(\rho)\right|+\alpha_{2} \Delta v(\rho)\right)
\end{gathered}
$$

and

$$
\Delta v(\rho)=\tanh \left(\alpha_{3} \frac{\rho}{\rho_{m}}\right)\left(u(\rho)+c \rho_{m}\left(\frac{1}{\rho}-\frac{1}{\rho_{m}}\right)\right),
$$

with parameters $a_{c}=2 \mathrm{~m} / \mathrm{s}^{2}, d_{c}=-5 \mathrm{~m} / \mathrm{s}^{2}, \hat{T}=0.1 \mathrm{~s}$, $\alpha_{1}=-0.2, \alpha_{2}=-0.8, \alpha_{3}=7$ and $c=-14 \mathrm{~km} / \mathrm{h}$. The density values, which determine the sign of $\beta$ according to Eqs. (5)-(6), are $\rho_{1}=19.09[1 / \mathrm{km} /$ lane $]$ and $\rho_{2}=\rho_{m}$. Throughout this work, we model two-lane sections of a highway without entries and exits with the above parameter set. In comparison to [16] theses parameters describe traffic flow more realistically, although we have not used experimental traffic data to determine them for a specific highway section. Note that the general conditions (5)(7) are sufficient to obtain multivalued fundamental diagrams. However, the quantitative details depend on the precise choice of $\beta$. As our simulations show, the analytically derived properties of the model of Aw, Rascle and Greenberg, that the velocity does not become negative and collisions do not occur, carry over to our system. For the numerical simulations of the model equations (11)-(2) we used a high-resolution shock-capturing scheme with an approximate Riemann solver. We chose a spatial resolution of $20 \mathrm{~m}$ and dynamically adapted the temporal resolution to half the value obtained from the Courant condition. The numerical method is described in detail in [16].

In order to obtain a deeper insight into the structure of the BVT model we study the smooth steady state solutions in Sec. II Our numerical simulations produce more general solutions approximating steady states solutions, which will be discussed in Sec. III In Sec. IV we assess the stability properties of the steady state solutions. With theses results we classify the traffic states of the BVT model according to the three traffic phases of Kerner [5] in Sec. $\mathbf{V}$ and apply the BVT model to simulate traffic flow at a bottleneck in Sec. VI We conclude the paper in Sec. VII

\section{SMOOTH STEADY STATE SOLUTIONS OF THE BVT MODEL}

For smooth solutions the balance equations describing traffic flow (1)-(2) can be rewritten as

$$
\begin{aligned}
\frac{\partial \rho}{\partial t}+v \frac{\partial \rho}{\partial x}+\rho \frac{\partial v}{\partial x} & =0 \\
\frac{\partial v}{\partial t}+\left(v+\rho u^{\prime}(\rho)\right) \frac{\partial v}{\partial x} & =\beta(\rho, v)(u(\rho)-v) .
\end{aligned}
$$

In the following, we study the smooth steady state solutions of the BVT model. In comparison to the study by Lee, Lee and Kim [19], our analysis - being performed on a first order system - is considerably simpler. Note, however, that due to the possibility of dealing with discontinuous solutions, we can in principle have more general solutions in the balanced system (11)-(2) (see also [1] and references therein).

Let us repeat, that for a steady state solution, there is a coordinate system $(\tilde{t}, z)$ and a constant velocity $w$

$$
\begin{aligned}
& x=z-w \tilde{t}, \\
& t=\tilde{t}
\end{aligned}
$$

such that

$$
\begin{aligned}
& \frac{\partial \rho}{\partial \tilde{t}}=0 \\
& \frac{\partial v}{\partial \tilde{t}}=0
\end{aligned}
$$

It follows from the continuity equation (13) that for all steady state solutions there is a constant $q$ such that

$$
\rho v=q+\rho w
$$

Hence steady state solutions are restricted to straight lines in the fundamental diagram of traffic flow. Moreover, the minimum and maximum speed of information propagation in system (1)-(2) limit the physically admissible steady state solutions, i.e. the velocity $w$, as

$$
\lambda_{1}=v+\rho u^{\prime}(\rho) \leq w \leq \lambda_{2}=v
$$

Let us assume that $v-w>0($ else $q=0)$. Then we can solve Eqn. (19) for the density

$$
\rho=\frac{q}{v-w}
$$


and substitute it into Eqn. (14), yielding the ordinary differential equation for steady state solutions in the BVT model

$$
\left(\lambda_{1}-w\right) \frac{d v}{d z}=\beta\left(\frac{q}{v-w}, v\right)\left(u\left(\frac{q}{v-w}\right)-v\right) .
$$

\section{A. Trivial steady state solutions}

We first study the trivial (constant) solutions of this ODE, i.e. solutions fulfilling $\frac{d v}{d z}=0$. The solutions are:

- The equilibrium velocity curve:

$$
v=v^{e}=u(\rho) .
$$

- The jam line (compare to the line J of Kerner [5]):

$$
\begin{array}{r}
v=v^{j}=u(\rho)+\left(\alpha_{1}+\alpha_{2}\right) \Delta v(\rho) \\
\text { for } \rho_{1}<\rho<\rho_{2} .
\end{array}
$$

Note that $\rho v<\rho u(\rho)$.

- The high-flow branch:

$$
\begin{array}{r}
v=v^{h}=u(\rho)+\left(\alpha_{1}-\alpha_{2}\right) \Delta v(\rho) \\
\text { for } \rho_{1}<\rho<\rho_{2} .
\end{array}
$$

Note that $\rho v>\rho u(\rho)$ in this case.

We summarize the trivial steady state solutions in Fig. 1 We further show in this figure the results of simulation runs for constant initial data in equilibrium $\rho=\rho_{0}$, $\rho_{0}=1,2, \ldots, 159[1 / \mathrm{km} /$ lane $], v=u\left(\rho_{0}\right)$ on a $7 \mathrm{~km}$ long stretch of a highway with periodic boundary conditions, prescribing a small amplitude perturbation of the density $\delta \rho=\sin (\pi x)$ initially located between 2 and $3 \mathrm{~km}$ on top. The data points were extracted from the simulations at 5 equidistantly distributed virtual detectors after an evolution time of $10 \mathrm{~h}$, without applying a temporal aggregation. As one can see from the plot many data points of the numerical solutions are closely related to branch sections of trivial steady state solutions, in particular for the jam line.

\section{B. Non-trivial steady state solutions}

Let us compare the equation of steady states (22) in analogy to the dynamics in classical mechanics presented in [19] to the following equation

$$
\left(\lambda_{1}-w\right) \frac{d v}{d z}=-\frac{d}{d v} U(v, w, q)
$$

with a potential energy $U$. We find that the potential $U(v, \ldots)$ has a functional form which is camelback-shaped

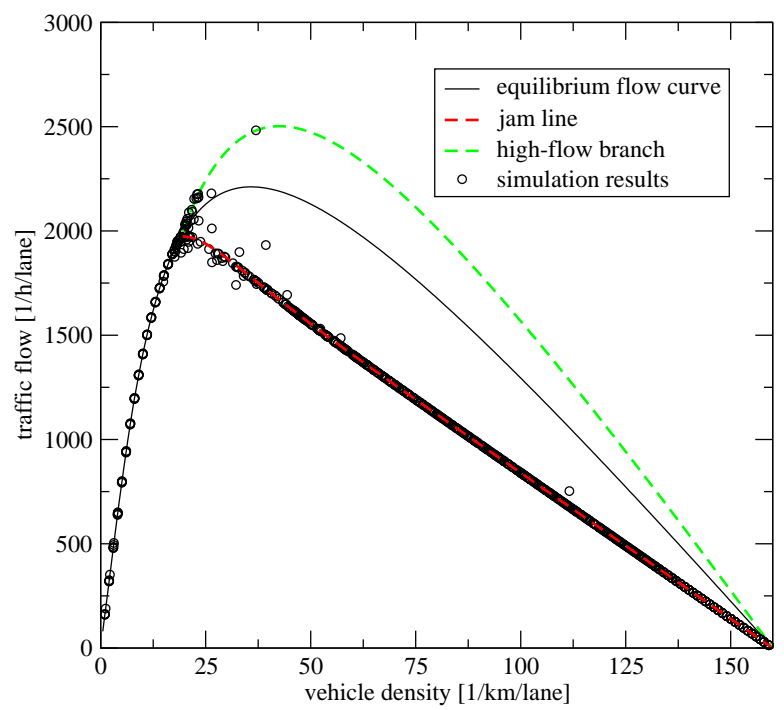

FIG. 1: (Color online) The trivial steady state solutions (i.e. solutions with $\left.\frac{d v}{d z}=0\right)$ of the BVT model. These solutions are the equilibrium solution $v=u(\rho)$ (solid black curve) and the two branches fulfilling $\beta(\rho, v)=0$ (dashed curves). Moreover, we present the results of simulation runs of perturbed equilibrium data. The simulated data points in the fundamental diagram are closely related to sections of trivial steady state solutions fulfilling $\beta(\rho, v)=0$.

for a wide range of constant values $w$ and $q$ as in [19]. However, unlike in 19], we do not obtain an acceleration term in our first order system. Note that according to (20) $\lambda_{1}-w \leq 0$.

In the following we restrict the discussion to the physically admissible smooth solutions (in particular we do not consider solutions with infinite gradient). In the limit $z \rightarrow \pm \infty$ all maximally extended steady state solutions approach one of the following curve sections:

A: free equilibrium flow: $v=u(\rho)$ and $\rho \leq \rho_{1}$,

B: unstable equilibrium flow: $v=u(\rho)$ and $\rho_{1}<\rho \leq \rho_{2}$,

C: jam line: see Eqn. (24),

D: high-flow branch: see Eqn. (25),

We classify the non-trivial maximally extended steady solutions according to the behavior in the limit $z \rightarrow \pm \infty$, using the letters of the corresponding branches. Due to the characteristic structure of the BVT model (see Fig. 2), only five solution classes can appear. These are the classes AD, BC, BD, CC and DD [26]. Schematically, all these steady state solutions have the form indicated in Fig. 3 i.e. solutions lying in the regions II and IV of Fig. 2 fulfill the condition $\frac{d v}{d z}>0$, solutions in regions I and III obey $\frac{d v}{d z}<0$ respectively and $\lim _{z \rightarrow \pm \infty} \frac{d v}{d z}=0$. Comparing our classification to the classification of Lee, Lee, Kim [19] for cases, where the parameters $q$ and $w$ 


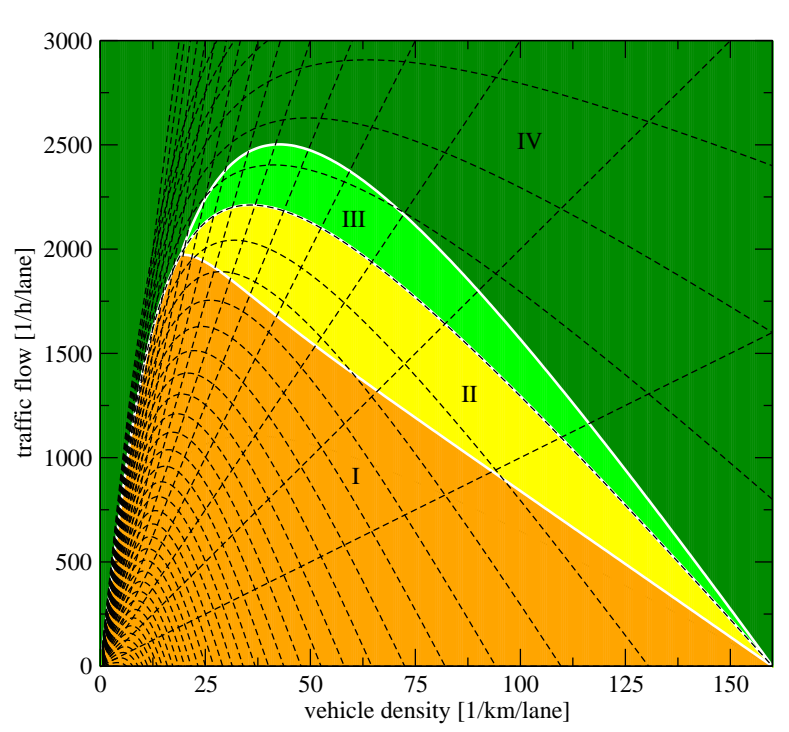

FIG. 2: (Color online) Characteristic structure and steady state solutions of the BVT model. As solid lines we plot the trivial steady state solutions bordering the regions I IV. On top (dashed curves) we plot the characteristic curves with slope $\lambda_{1}$ and $\lambda_{2}$ respectively. As the speed of steady state solutions is limited by the characteristic speeds $\lambda_{1}$ and $\lambda_{2}$, physically admissible steady state solutions lie inside the characteristic cones spanned by these two speeds at every point $(\rho, \rho v)$ in the flow-density diagram.

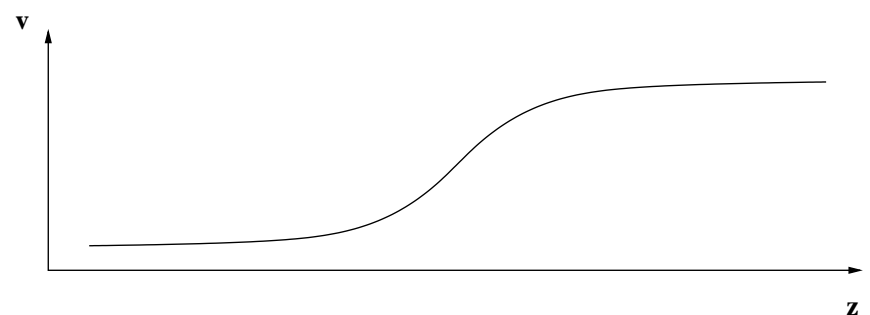

FIG. 3: Sketch of the non-trivial steady state solutions. The steady state solutions fulfill $\frac{d v}{d z}>0$ (or $\frac{d v}{d z}<0$ respectively) and $\lim _{z \rightarrow \pm \infty} \frac{d v}{d z}=0$.

lead to a camelback-shaped profile of the potential $U$, the solutions correspond to minimum-saddle solutions (without oscillations). The possible appearance of the different solution classes in the flow-density diagram is shown in Fig. 4 .

\section{QUASI STEADY STATE SOLUTIONS}

In principal, some of the non-trivial steady state solutions described before can be glued together to form discontinuous, periodic steady state solutions 14, 15]. Steady state solutions can be linked by a shock wave, if the quantities $\rho_{-}, v_{-}$left to the interface and the corre-
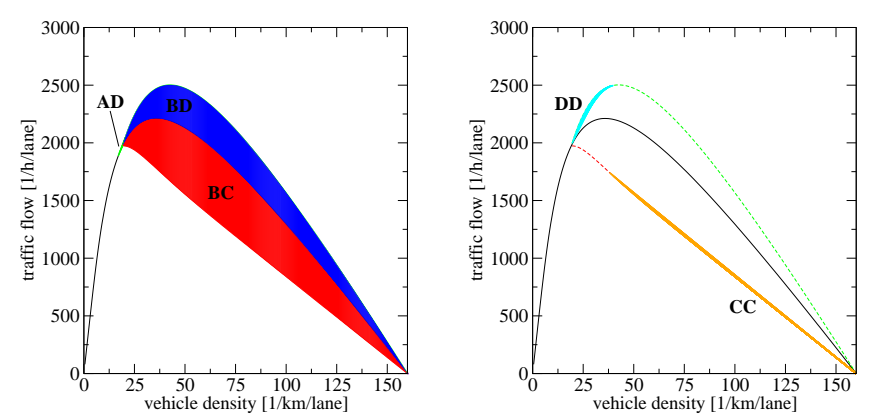

FIG. 4: (Color online) Left Panel: Regions covered by the smooth maximally extended non-trivial steady state solutions linking different branches, which are consistent with the characteristic structure of the BVT model. The solutions link the free equilibrium flow solution with the high-flow branch (class AD), the unstable equilibrium solution with the jam line (class $\mathrm{BC}$ ) and the unstable equilibrium solution with the high-flow branch (class BD).

Right Panel: Regions covered by the smooth maximally extended non-trivial steady state solutions linking identical branches, which are consistent with the characteristic structure of the BVT model. For class CC the non-trivial steady states link data points lying on the jam line, for the class DD they link data points of the high-flow branch.

sponding quantities $\rho_{+}, v_{+}$right of the interface satisfy the following conditions [9, 11]:

$$
\begin{gathered}
\rho_{+}>\rho_{-}, \\
\rho_{+}\left(v_{+}-u\left(\rho_{+}\right)\right)=\rho_{-}\left(v_{-}-u\left(\rho_{-}\right)\right) .
\end{gathered}
$$

With the equation of steady state solutions

$$
\rho_{ \pm} v_{ \pm}=q+\rho_{ \pm} w
$$

we obtain from Eqn. (28)

$$
w\left(\rho_{+}-\rho_{-}\right)=\rho_{+} u\left(\rho_{+}\right)-\rho_{-} u\left(\rho_{-}\right) .
$$

The monotonicity of the velocity of steady state solutions, which follows from Eqn. (22) (see also Fig. 31), relates to the monotonicity of the density according to

$$
\frac{d \rho}{d z}=-\frac{\rho}{v-w} \frac{d v}{d z} .
$$

Therefore, condition (27) restricts periodic steady state solutions linked by shock waves to regions II and IV of Fig. 2

In the following we focus on steady state solutions of type CC in region II. In particular, steady state solutions lying inside region II have to approach the jam line at velocity values for which the condition

$$
\left(\rho v^{j}(\rho)\right)^{\prime \prime}>0
$$


is fulfilled. For the chosen parameter values, this implies $\rho_{-}>36.51[1 / \mathrm{km} /$ lane $]$. A necessary requirement to fulfill Eqn. (30) is the condition

$$
\left.(\rho u(\rho))^{\prime}\right|_{\rho=\rho_{-}} \geq\left(\rho v^{j}(\rho)\right)_{\rho=\rho_{-}}^{\prime},
$$

which results in our particular case to $\rho_{-} \leq 61.57$ $[1 / \mathrm{km} /$ lane]. Therefore, for the chosen parameter values there is only a very small parameter range where periodic steady state solutions of type CC linked by shock waves are possible. However, in our numerical simulation, we find quasi steady state solutions in a much larger region of parameter space (see Fig. 1). To obtain a more detailed
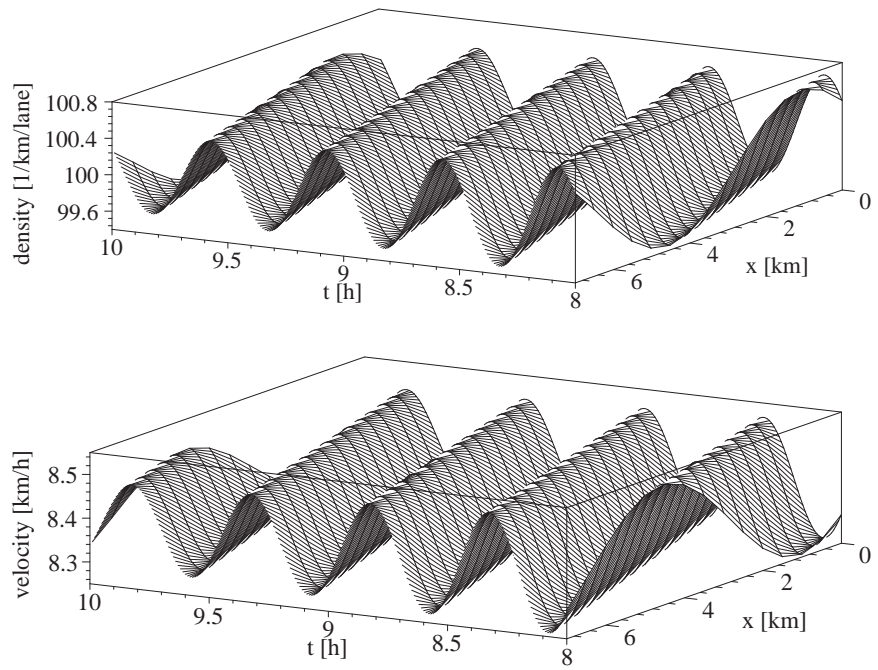

FIG. 5: Quasi steady state solution at the jam line for a numerical simulation of perturbed equilibrium data with initial density $\rho=100[1 / \mathrm{km} /$ lane $]$. Between a simulation time of 8 and $10 \mathrm{~h}$ the evolution is quasi-stationary with a propagation speed of about $w \approx-14 \mathrm{~km} / \mathrm{h}$.

picture of these quasi stationary solutions, we plot the simulation results of unstable equilibrium data in Fig. 5. focusing on the evolution between 8 and 10 hours simulation time. We started the simulation of Fig. 5] with constant initial data $\rho=\rho_{0}=100[1 / \mathrm{km} /$ lane $], v=u\left(\rho_{0}\right)$ and a sinusoidal density perturbation on top. After a long evolution time we obtain a quasi steady state close to the jam line, propagating with a velocity $w \approx-14$ $\mathrm{km} / \mathrm{h}$ upstream. This quasi steady state solution is not a true steady state solution, the amplitude slowly decreases with time, as it can be scarcely noted in the figure. The solution consists of different branches close to the trivial steady state solutions lying in regions I/II of Fig. 2 Thus, for quasi steady state solutions the quantities $q$ and $w$ of steady state solutions (see Eqn. (19)) are only approximately constant.

Similar quasi-steady state solutions also exist for a section of the high-flow branch (see Sec. IV below). They consist of approximate steady state solutions lying in regions III/IV of Fig. 2] These quasi steady state solutions travel downstream with a velocity approximately given by the tangent of the high-flow branch.

\section{STABILITY ANALYSIS OF THE STEADY STATE SOLUTIONS}

Due to the importance of steady state solutions at the jam line and the high-flow branch (see Fig. 11) we focus the stability analysis on the trivial steady state solutions of the BVT model. In principal the code can be applied to study the stability of the non-trivial solutions as well, although more accurate results may be obtained using specialized methods to this aim (see e.g. [20]).

\section{A. Linear stability analysis}

As presented in [16] the equilibrium flow curve $\rho v=$ $\rho u(\rho)$ is linearly stable for $\rho<\rho_{1}$ and linearly unstable in the intermediate to high density regime $\rho_{1}<\rho<$ $\rho_{2}$. Here we extend the linear stability analysis to all steady state solutions obtained from setting $\beta(\rho, v)=$ 0, i.e. steady state solutions Eqs. (24) and (25). We denote the corresponding constant states $\left(\rho_{0}, v^{j / h}\left(\rho_{0}\right)\right)$. Plugging the ansatz

$$
\begin{aligned}
& \rho=\rho_{0}+\tilde{\rho} \exp (i l x+\omega(l) t), \\
& v=v^{j / h}\left(\rho_{0}\right)+\tilde{v} \exp (i l x+\omega(l) t)
\end{aligned}
$$

into the evolution equations (13) and (14) we obtain as characteristic equations for the existence of solutions $(\tilde{\rho}, \tilde{v}) \neq(0,0)$ :

$$
\begin{aligned}
\left(\omega+i l v^{j / h}\right)^{2}+ & \left(\omega+i l v^{j / h}\right)\left(i l u^{\prime} \rho_{0}+\left(v^{j / h}-u\right) \frac{\partial \beta}{\partial v}\right) \\
& -\rho_{0} i l \frac{\partial \beta}{\partial \rho}\left(v^{j / h}-u\right)=0
\end{aligned}
$$

Solving the last equation for $\omega$ we can distinguish between the linearly stable and unstable regime of the trivial steady-state solutions, i.e. curve sections with $\operatorname{Re}(\omega) \leq 0$ for arbitrary $l$ and curve sections for which $\operatorname{Re}(\omega)>0$ for some $l$ respectively. We find that the jam line is linearly unstable for $\rho_{1}<\rho \leq \tilde{\rho}_{j}=39.73$ $[1 / \mathrm{km} /$ lane $]$ and linearly stable for $\tilde{\rho}_{j}<\rho<\rho_{2}$, whereas the high-flow branch is linearly stable for densities $\rho_{1}<$ $\rho \leq \tilde{\rho}_{h}=39.73[1 / \mathrm{km} /$ lane $]$ and linearly unstable for $\tilde{\rho}_{h}<\rho<\rho_{2}$.

In the following we will give a more intuitive explanation for the above results. For trivial steady state solutions with exactly constant density and velocity profile, the characteristic structure (20) does not give any restrictions. However, quasi steady state solutions with nonconstant density (velocity) on these trivial steady state branches travel with a velocity $w$ which corresponds to the derivative of the flow density curve

$$
w \approx \frac{d\left(\rho v^{j / h}\right)}{d \rho}
$$

Hence, according to condition (20) the local characteristic cone has to enclose the corresponding steady state 
branch spanned by the characteristic speeds $\lambda_{1}$ and $\lambda_{2}$, i.e. $\lambda_{1} \leq w \leq \lambda_{2}$. For our parameter values the characteristic condition restricts possible stable (quasi) steady state solutions at the jam line to solutions fulfilling $\rho>\tilde{\rho}_{j}=39.73[1 / \mathrm{km} /$ lane $]$ and those at the high-flow branch to solutions fulfilling $\rho \leq \tilde{\rho}_{h}=39.73[1 / \mathrm{km} /$ lane $]$ (see Fig. 2). Note, that the two densities need not agree for a general parameterization. For our parameterization, it follows from the appearance of the term $\Delta v(\rho)$ in both, Eqs. (24) and (25).

Next we study the nonlinear stability properties in simulations of the full system.
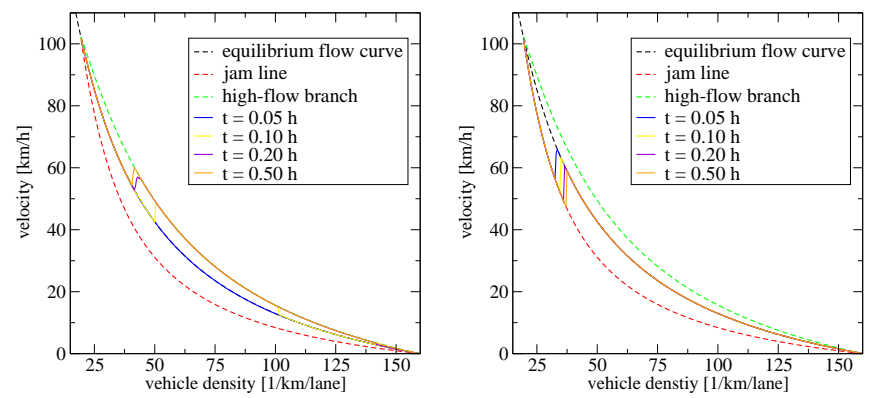

FIG. 6: (Color online) Left panel: Stability properties of the high-flow branch. We study the stability properties by prescribing constant steady state solutions $\rho=\rho_{0}, v=$ $v^{h}\left(\rho_{0}\right)$ and on top a sinusoidal velocity perturbation $\delta v=$ $v_{\text {ampl }} \sin (\pi x)$ for $2<x<3 \mathrm{~km}$. In the figure, we plot the maximum value of $v^{h}\left(\rho_{0}\right)+v_{\text {ampl }}, v_{\text {ampl }} \leq 0$, for which the initial data is unstable against the formation of synchronized flow, for different evolution times. For densities $\rho<40$ $[1 / \mathrm{km} /$ lane $]$, the high-flow branch is metastable, it becomes unstable against the formation of synchronized flow for sufficiently small values $v_{\text {ampl }}$. For densities $\rho>40[1 / \mathrm{km} /$ lane] the high-flow branch is unstable against the formation of synchronized traffic flow.

Right panel: Analysis of the stability properties of the jam line using constant steady state solutions $\rho=\rho_{0}, v=$ $v^{j}\left(\rho_{0}\right)$ and on top a sinusoidal velocity perturbation $\delta v=$ $v_{\text {ampl }} \sin (\pi x)$ for $2<x<3 \mathrm{~km}$. In the figure, we plot the minimum value of $v^{j}\left(\rho_{0}\right)+v_{\text {ampl }}, v_{\text {ampl }} \geq 0$, for which the initial data is unstable against the formation of synchronized flow, for different evolution times. For densities $\rho>40$ [1/km/lane] the jam line is metastable, it becomes unstable only for sufficiently large values $v_{\text {ampl }}$. For densities $\rho<40[1 / \mathrm{km} /$ lane] the jam line is unstable against the formation of synchronized traffic flow.

\section{B. Numerical results}

We first analyze the stability properties of the highflow branch of steady state solutions, Eqn. (25). To this aim, we use constant steady state initial data $\rho=\rho_{0}, v=$ $v^{h}\left(\rho_{0}\right)$ with a sinusoidal perturbation $\delta v=v_{\text {ampl }} \sin (\pi x)$ for $2<x<3 \mathrm{~km}$, prescribing periodic boundary conditions on a $7 \mathrm{~km}$ long highway. In order to decide whether synchronized flow appears during the numerical evolution, we use the criteria $v(\rho)<u(\rho)$ or $v(\rho)<$ $v^{h}(\rho)-\left|v_{\text {ampl }}\right|$.

As our analysis shows, the stability properties of the high-flow branch depend on the particular perturbation. For the density regime $\rho_{1}<\rho \leq \tilde{\rho}_{h}$, the high-flow branch is metastable against the formation of synchronized flow, i.e. for small amplitude perturbations, no synchronized flow appears, whereas for larger velocity perturbations with negative amplitude $v_{\text {ampl }}$, synchronized flow appears. For the density regime $\tilde{\rho}_{h}<\rho<\rho_{2}$, the high-flow branch is unstable against the formation of synchronized flow. We summarize the corresponding results in the left panel of Fig. 6

Second, we study the stability properties of the jam line, using constant steady state initial data $\rho=\rho_{0}$, $v=v^{j}\left(\rho_{0}\right)$ again with a sinusoidal perturbation $\delta v=$ $v_{\text {ampl }} \sin (\pi x)$ for $2<x<3 \mathrm{~km}$. In this case, we use the criteria $v(\rho)>u(\rho)$ or $v(\rho)>v^{j}(\rho)+\left|v_{\text {ampl }}\right|$ to identify synchronized flow.

For densities $\tilde{\rho}_{j}<\rho<\rho_{2}$ the jam line is metastable against the formation of synchronized flow, i.e. for small amplitude perturbations, the jam line is stable, for larger amplitude perturbations (with positive amplitude $v_{\text {ampl }}$ ), synchronized flow appears. In contrast, for densities $\rho_{1}<\rho \leq \tilde{\rho}_{j}[1 / \mathrm{km}]$ the jam line is unstable against the formation of synchronized flow. We show the results in the right panel of Fig. [6]

We summarize the results of the stability analysis in Fig. 17 The figure shows the observed gap between free and congested flow in the fundamental diagram of traffic flow separating the (meta-) stable branch sections.

\section{IDENTIFICATION OF KERNER'S THREE PHASES OF TRAFFIC FLOW}

Kerner [5, 21, 22, 23] classifies traffic flow into three phases: free flow, synchronized flow and wide moving jams. In this section we try to relate the traffic states of the BVT model to Kerner's three phases. We will first summarize our classification, before we will discuss the motivation.

- free flow: Steady state solutions at the equilibrium velocity curve $u=u(\rho)$ for the density regime $0 \leq$ $\rho \leq \rho_{1}$ (free equilibrium flow) and (quasi) steady state solutions close to the high-flow branch in the metastable regime $\rho_{1}<\rho \leq \tilde{\rho}_{h}$ make up the free flow state.

- wide moving jams: Spatially extended (quasi) steady state solutions at the jam line in the metastable regime $\tilde{\rho}_{j}<\rho<\rho_{2}$ make up wide moving jams.

- synchronized flow: All other congested traffic states including the non-trivial steady state solutions of type $\mathrm{BC}$ and $\mathrm{BD}$ form synchronized traffic flow. 


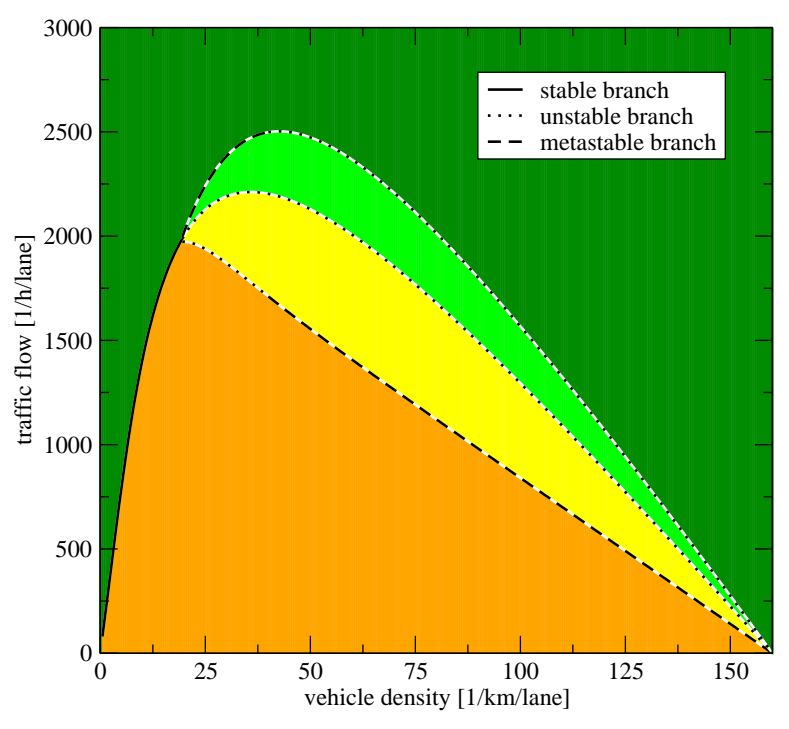

FIG. 7: (Color online) Results of the stability analysis of the trivial steady state solutions $\frac{d v}{d z}=0$. Curve sections represented as solid black line correspond to the linearly stable steady state solutions. Curve sections represented as dotted line and dashed line correspond to unstable and metastable steady state solutions respectively.

\section{A. Free flow}

For small densities $\left(0 \leq \rho \leq \rho_{1}\right)$ free flow is stable in the BVT model. Moreover, the model can reproduce the metastability of free flow against the formation of synchronized flow (see Fig. 6), which is observed for traffic states at the high-flow branch $\rho_{1}<\rho \leq \tilde{\rho}_{h}$. In the model instabilities only appear for velocity perturbations with negative amplitude.

\section{B. Wide moving jams}

As our results show wide moving jams as defined above are stable against small amplitude perturbations. For the chosen parameter values the propagation speed of wide moving jams lies between $-16<w<-14[\mathrm{~km} / \mathrm{h}]$ for the density region $\tilde{\rho}_{t}<\rho \leq \rho_{2}$, i.e. it is nearly constant and reproduces the observed value.

We further analyzed the outflow from wide moving jams (see Fig. 8). We find for wide moving jams in the density region $\tilde{\rho}_{t}<\rho<125[1 / \mathrm{km} /$ lane $]$ a constant outflow of about $f_{\text {out }}=1914[1 / \mathrm{h} /$ lane $]$. This can be seen in the lower right panel of Fig. 8] where we plot the outflow from wide moving jams $\rho=\rho_{j}, v=v^{j}\left(\rho_{j}\right)$ initially located between 2 and $3 \mathrm{~km}$ and surrounded by a region of free flow $\rho=\rho_{f}, v=u\left(\rho_{f}\right)$.
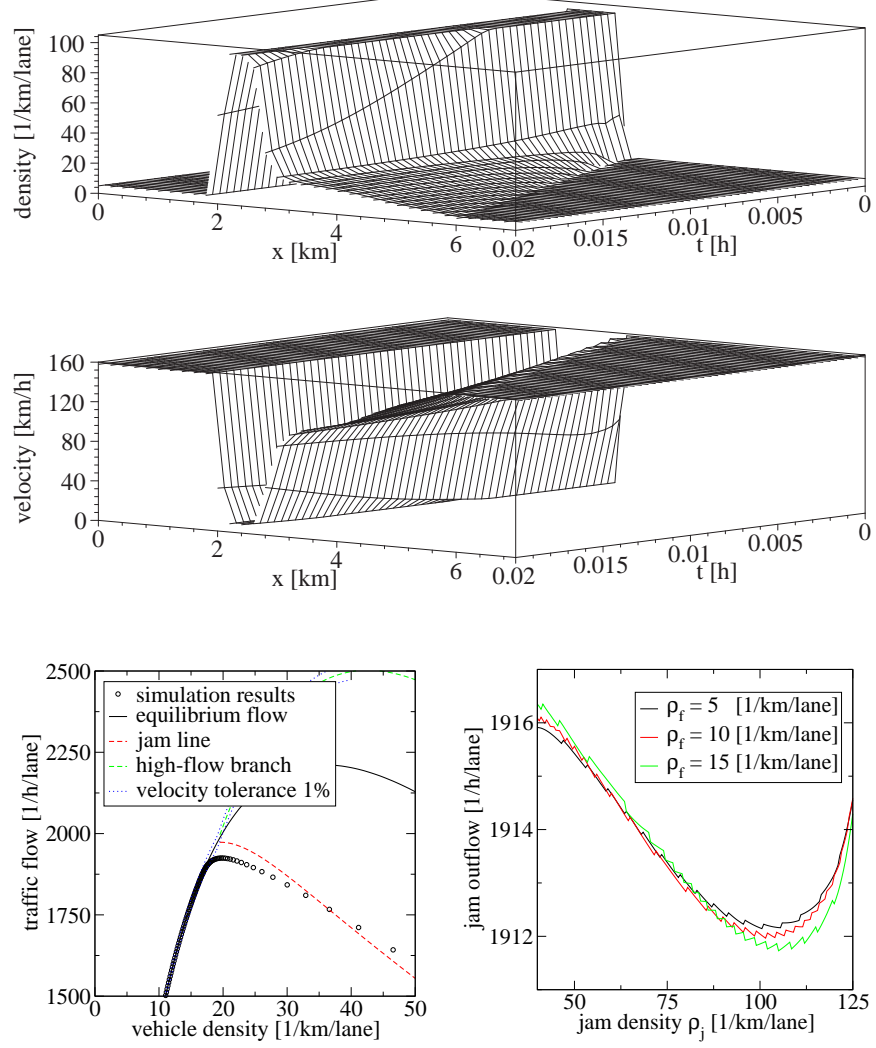

FIG. 8: (Color online) Upper two panels: Simulation of the outflow from a wide moving jam. Plotted are the evolution of the density and the velocity as a function of space and time. In the initial data we prescribe a wide moving jam with density $\rho_{j}=100[1 / \mathrm{km} /$ lane $]$ between 2 and $3 \mathrm{~km}$, and free flow with density $\rho_{f}=5[1 / \mathrm{km} /$ lane $]$ elsewhere. During the evolution, the wide moving jam narrows down and finally dissolves. Lower left panel: Flow-density diagram for the above simulation results after $t=0.02 \mathrm{~h}$. In addition we plot the curve representing the equilibrium flow as well as the jam line and the high-flow branch. We determine the outflow of the wide moving jam at that point for which the velocity of the outflow differs from the equilibrium velocity by less than $1 \%$.

Lower right panel: Outflow from wide moving jams, varying the jam density $\rho_{j}$ of the wide moving jams between 2 and 3 $\mathrm{km}$. The three different curves correspond to different values of free flow density $\rho_{f}$ in the region between 0 and $2 \mathrm{~km}$ and 3 and $7 \mathrm{~km}$. As one can see from the plot, the outflow from the wide moving jams only varies within a very small range of flow values and it is largely independent of the density of free flow. The typical outflow from wide moving jams for the chosen parameter values is $f_{\text {out }} \approx 1914$ [1/h/lane], which is far below the maximum of metastable free flow $f=2487$ $[1 / \mathrm{h} /$ lane $]$.

\section{Synchronized flow}

Synchronized flow as defined above covers a wide region in the fundamental diagram. This can be already seen in the left panel of Fig. 4 for steady state solutions of type $\mathrm{BC}$ and $\mathrm{BD}$. We exemplarily show the 
formation of synchronized flow from free flow of density $\rho=30[1 / \mathrm{km} /$ lane $]$. As a nucleus for the emergence of synchronized flow, we use a velocity perturbation $\delta v=-7 \sin (\pi x)$ located between 2 an $3 \mathrm{~km}$ on a highway with periodic boundary conditions. The evolution of these initial data leads to states which are widely scattered in the fundamental diagram, as it can be seen in Fig. 9] We remark that in an earlier work 24] the scattering was reproduced by modeling different vehicle types. Here, in contrast, the scattering already follows from the traffic dynamics without distinguishing between different vehicle types. Including different vehicle types into the BVT model, which is beyond the scope of the current work, would be expected to further widen the scattering.

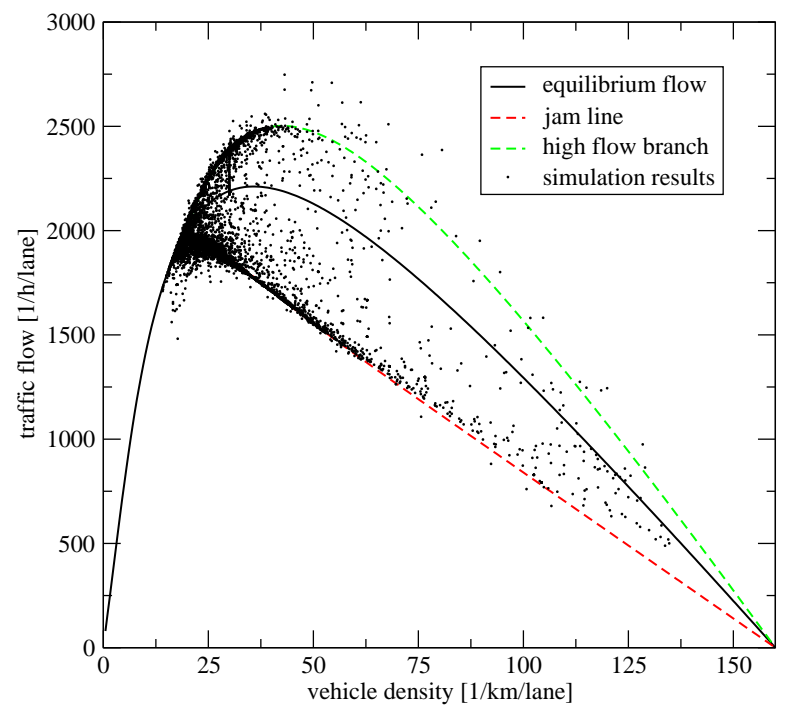

FIG. 9: (Color online) Formation of synchronized flow from metastable free flow of density $\rho=30[1 / \mathrm{km} /$ lane $]$ with an initial velocity perturbation $\delta v=-7 \sin (\pi x)$ located between 2 an $3 \mathrm{~km}$. Due to the velocity perturbation, the free flow state breaks down, leading to a complicated pattern of synchronized flow which covers a wide region of states in the fundamental diagram. Finally, moving jams form, which can in turn lead to free flow of lower density, thus reproducing the hysteresis effect observed in traffic dynamics. The plot shows all data points corresponding to the constant time slices at $t=i \Delta t$, where $\Delta t=0.1 \mathrm{~h}, i=0, . ., 50$.

\section{TRAFFIC FLOW AT BOTTLENECKS}

In this section we study the behavior of traffic flow in the BVT model at a highway bottleneck. We focus the discussion on two simulation runs of a two-lane highway with periodic boundary conditions [27]. Again, we use a longitudinal extension of the highway of $7 \mathrm{~km}$ with homogeneous initial free flow of density $\rho=37.5[1 / \mathrm{km} /$ lane $]$. We model the bottleneck simply by a velocity modification (velocity drop for free flow) between 5 and $6 \mathrm{~km}$, setting the velocity to a modified value $v^{\text {mod }}$ after each numerical evolution step,

$$
v^{\bmod }=v+(u(\rho)-v-0.1 \mathrm{~km} / \mathrm{h})|\sin (\pi x)|
$$

We show the numerical evolution in Fig. 10. Despite
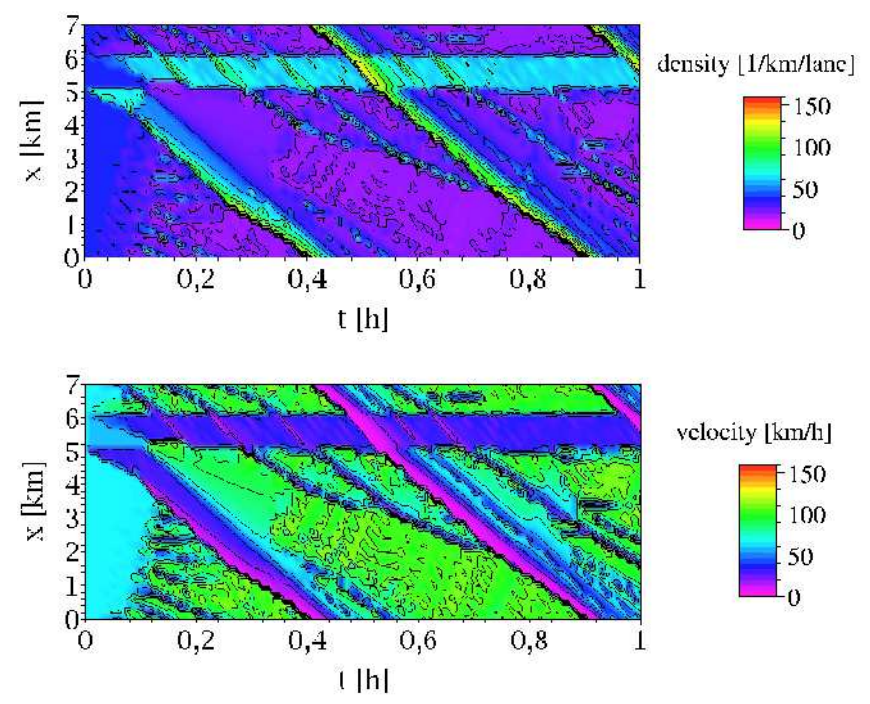

FIG. 10: (Color online) Formation and propagation of wide moving jams. The upper panel shows the evolution of the density, whereas the lower panel shows the evolution of the velocity. At the bottleneck located between 5 and $6 \mathrm{~km}$, synchronized flow forms, which finally leads to a wide moving jam. This wide moving jam moves with a velocity of about $-15 \mathrm{~km} / \mathrm{h}$ (i.e. upstream) and swallows moving jams during this propagation. It further travels through the bottleneck. See the text for a detailed description.

the simplicity of the initial setup the numerical evolution shows a very complicated dynamics. As we will discuss below, we observe the formation of synchronized flow and wide moving jams.

As a consequence of the bottleneck, the initial velocity drops to smaller values in the bottleneck region (dark blue regions between 5 and $6 \mathrm{~km}$ in the velocity plot), but also further upstream (dark blue regions between 4 and $5 \mathrm{~km}$ at about 0.1h). Both regions correspond to synchronized flow. The first synchronized flow region stays fixed at the bottleneck, however the upstream front can oscillate in time (e.g. between $0.8 \mathrm{~h}$ and $0.9 \mathrm{~h}$ ). The second region of synchronized flow travels upstream. It takes some time until an accentuated wide moving jam with velocities close to zero forms. This wide moving jam travels further upstream and reenters the numerical domain at $7 \mathrm{~km}$ after $t \approx 0.4 \mathrm{~h}$ due to the periodic boundary conditions used in the numerical simulation. When reaching the bottleneck, it simply travels through the first synchronized region, thus becoming a foreign 
wide moving jam. Note, that the velocity of the downstream front of this wide moving jam is nearly constant and has a value of about $-15 \mathrm{~km} / \mathrm{h}$.

Between the wide moving jams we observe regions of low density and high velocity, which correspond to free flow (see e.g. the region at $x=1 \mathrm{~km}$ for $t=0.7 \mathrm{~h}$ ), and smaller moving jams. As one can see from the plot there are several regions (pinch regions) where these additional moving jams form (see e.g. the region between 0 and 3 $\mathrm{km}$ for $t \approx 0.15 \mathrm{~h}$ or at about $1.5 \mathrm{~km}$ for $t \approx 0.45 \mathrm{~h}$ ). For these moving jams, the downstream front is in general not as robust as for the wide moving jam described above. At $x=3.5 \mathrm{~km}$ for $t=0.55 \mathrm{~h}$, we observe the merging of two moving jams, which are finally swallowed by the wide moving jam at about $x=2 \mathrm{~km}$ for $t=0.75 \mathrm{~h}$. We also observe an example for the catch effect of a narrow moving jam, see the region at $x=5.5 \mathrm{~km}$ for $t \approx 0.15 \mathrm{~h}$.
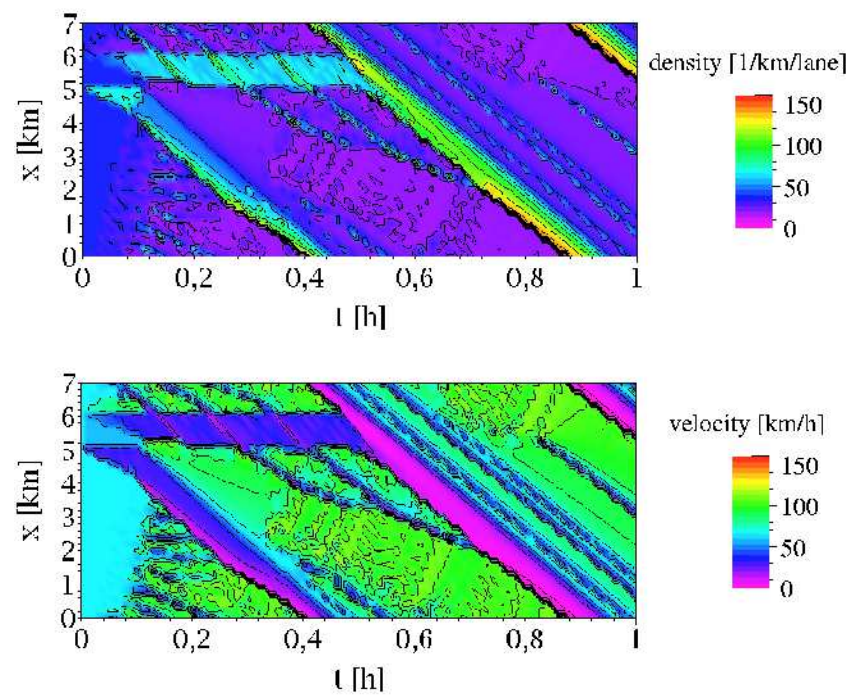

FIG. 11: (Color online) Formation and propagation of wide moving jams. The simulation setup is identical to that of Fig. 10 except that after a time of $0.5 \mathrm{~h}$ the bottleneck is not effective any longer. As a consequence, the synchronized flow region pinned to the bottleneck region disappears, the corresponding wide moving jam becomes wider at late times.

Figure 11 shows the simulation results for the same initial setup, except that the bottleneck Eqn. (38) is only effective for simulation times $t<0.5 \mathrm{~h}$. As a consequence the synchronized flow region pinned to the bottleneck disappears at later times. The wide moving jam traveling through the former bottleneck region expands and reaches velocities close to zero inside the jam. This can be also seen in Fig. 12 where we plot the time series of the flow rate and the velocity for a detector located at $x=0 \mathrm{~km}$.
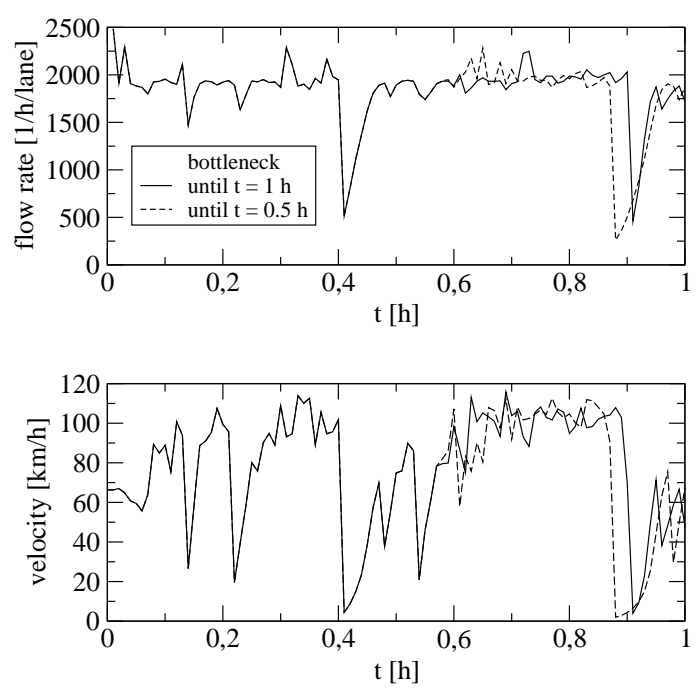

FIG. 12: Time series of the flow rate (upper panel) and the velocity (lower panel) as measured by a detector at location $x=0 \mathrm{~km}$ for the simulation results of Figs. 10 and 11 Inside the wide moving jam, small values of the traffic flow and in particular the velocity are reached.

\section{DISCUSSION}

The BVT model is a macroscopic, deterministic model, which describes vehicular traffic flow using standard methods from continuum fluid dynamics. It uses an equilibrium flow-density curve. In contrast to earlier models, however, the parameter range of the effective relaxation coefficient is extended to negative values. As a consequence, the equilibrium flow curve does not describe traffic states in the congested regime directly, but still determines the characteristic structure of the model. An additional consequence of the negative effective relaxation coefficient is the appearance of (two) additional branches of trivial steady states. The characteristic structure, i.e. the finiteness of propagation speeds, restricts the stability of these steady state solutions. The high-flow branch is metastable against the formation of synchronized flow for intermediate densities and unstable for high densities. We interpret the metastable section of high-flow branch as metastable free flow. Stable free flow, in contrast, corresponds to the stable equilibrium flow. The low flow branch in the congested regime (i.e. the jam line) is unstable against the formation of synchronized flow for intermediate densities and metastable for high densities. We interpret spatially extended solutions at the metastable branch of the jam line as wide moving jams. We further identify the unstable sections of the high-flow branch and the jam line, as well as the additional (steady state) solutions in the congested regime, 
which can lead to very complicated oscillatory patterns, as synchronized flow. Thus, synchronized flow covers a wide region of congested states in the fundamental diagram, without distinguishing between different vehicle types and driver characteristics in the model.

There are some additional results supporting the BVT model. In particular, the model ensures that wide moving jams do not form spontaneously from free flow. When the velocity drops below the critical value in free flow, the velocity is driven to even smaller values at that location which results in a strong gradient in the velocity. It is only after complicated oscillations have occurred, which lead to a rearrangement of the density and the velocity, that an extended steady state solution close to the jam line, i.e. a wide moving jam, appears.

We further can reproduce the characteristic properties

[1] R. Kühne and P. Michalopoulos, in Transportation Research Board special report 165, (5)1 (1997, available online at http://www.tfhrc.gov/its/tft/tft.htm).

[2] D. Helbing, Verkehrsdynamik (Springer, Berlin, 1997).

[3] D. Helbing, A. Hennecke, V. Shvetsov, and M. Treiber, Transportation Research Part B 35, 183 (2001).

[4] D. Helbing, Reviews of Modern Physics 73, 1067 (2001).

[5] B. Kerner, The Physics of Traffic (Springer, Berlin, 2004).

[6] F. John, Partial Differential Equations (Springer, New York, 1982).

[7] M. Lighthill and G. Whitham, Proceedings of the Royal Society A 229, 317 (1955).

[8] P. Richards, Operations Research 4, 42 (1956).

[9] A. Aw and M. Rascle, SIAM Journal on Applied Mathematics 60, 916 (2000).

[10] P. DuChateau and D. Zachmann, Applied Partial Differential Equations (Dover, Mineola, 2002).

[11] J. Greenberg, SIAM Journal on Applied Mathematics 62, 729 (2001).

[12] M. Rascle, Mathematical and Computer Modelling 35, 581 (2002).

[13] W. Jin and H. Zhang, Proceedings of the TRB Annual Meeting, Washington DC (2003).

[14] J. Greenberg, A. Klar, and M. Rascle, SIAM Journal on Applied Mathematics 63, 818 (2003).

[15] J. Greenberg, SIAM Journal on Applied Mathematics 64, 1175 (2004). of real wide moving jams with our model. For the chosen parameter values, the downstream front of wide moving jams travels upstream with a nearly constant velocity of about $15 \mathrm{~km} / \mathrm{h}$. Moreover, the outflow from wide moving jams is largely independent of the characteristics of the wide moving jam. We obtain a typical outflow of 1914 vehicles/h/lane. Furthermore, we showed, that wide moving jams travel through bottlenecks, whereas smaller moving jams can be caught by a bottleneck.

\section{Acknowledgments}

We thank James Greenberg for encouraging comments.
[16] F. Siebel and W. Mauser, SIAM Journal on Applied Mathematics 66, 1150 (2006).

[17] M. Koshi, M. Iwasaki, and I. Ohkura, in Proceedings of the 8th International Symposium on Transportation and Traffic Theory (V. Hurdle, E. Hauer, G. Stuart ed., 1983), pp. 403-424.

[18] G. Newell, Operations Research 9, 209 (1961).

[19] H. Lee, H.-W. Lee, and D. Kim, Phys. Rev. E 69, 016118 (2004).

[20] F. Bouchut, Nonlinear Stability of Finite Volume Methods for Hyperbolic Conservation Laws and Well-Balanced Schemes for Sources (Birkhäuser, Basel, 2004).

[21] B. Kerner and H. Rehborn, Phys. Rev. E 53, R4275 (1996).

[22] B. Kerner, Physical Review Letters 81, 3797 (1998).

[23] B. Kerner, Phys. Rev. E 65, 046138 (2002).

[24] M. Treiber and D. Helbing, J. Phys. A 32, L17 (1999).

[25] The system studied in this paper is a first order system consisting of two equations, see Eqs. (11)-(2).

[26] For the parameters used in [16], for which $\rho_{2}<\rho_{m}$, we would have an additional branch section (E) of stopped equilibrium flow $v=u(\rho)$ for $\rho_{2}<\rho \leq \rho_{m}$ and a sixth solution class DE.

[27] Using periodic boundary conditions enables us to study the propagation of moving jams through a bottleneck modeling only a single bottleneck. 